\title{
Birth Outcomes for Pregnant Women with HIV Using Tenofovir- Emtricitabine
}

\author{
Kathryn Rough, Sc.D., George R. Seage III, D.Sc., M.P.H., Paige L. Williams, Ph.D., Sonia \\ Hernandez-Diaz, M.D., Dr.P.H., Yanling Huo, M.S., Ellen G. Chadwick, M.D., Judith S. Currier, \\ M.D., Risa M. Hoffman, M.D., Emily Barr, C.P.N.P., C.N.M., M.S.N., David E. Shapiro, Ph.D., \\ Kunjal Patel, D.Sc., M.P.H., and for the PHACS and the IMPAACT P1025 Study Teams \\ Departments of Epidemiology (K.R., G.R.S., P.L.W., S.H.-D., K.P.) and Biostatistics (P.L.W., \\ D.E.S.) and the Center for Biostatistics and AIDS Research (Y.H., D.E.S., K.P.), Harvard T.H. \\ Chan School of Public Health, and the Division of Pharmacoepidemiology and Pharmaco- \\ economics, Department of Medicine, Brigham and Women's Hospital and Harvard Medical \\ School (K.R.), Boston; the Department of Pediatrics, Northwestern University Feinberg School of \\ Medicine, Chicago (E.G.C.); the Department of Medicine and Division of Infectious Diseases, \\ David Geffen School of Medicine at the University of California, Los Angeles, Los Angeles (J.S.C., \\ R.M.H.); and the Department of Pediatrics, University of Colorado School of Medicine, Aurora \\ (E.B.)
}

\section{Abstract}

BACKGROUND—In a previous trial of antiretroviral therapy (ART) involving pregnant women with human immunodeficiency virus (HIV) infection, those randomly assigned to receive tenofovir, emtricitabine, and ritonavir-boosted lopinavir (TDF-FTC-LPV/r) had infants at greater risk for very premature birth and death within 14 days after delivery than those assigned to receive zidovudine, lamivudine, and ritonavir-boosted lopinavir (ZDV-3TC-LPV/r).

METHODS—Using data from two U.S.-based cohort studies, we compared the risk of adverse birth outcomes among infants with in utero exposure to ZDV-3TC-LPV/r, TDF- FTC-LPV/r, or TDF-FTC with ritonavir-boosted atazanavir (ATV/r). We evaluated the risk of preterm birth $(<37$ completed weeks of gestation), very preterm birth ( $<34$ completed weeks), low birth weight $(<2500 \mathrm{~g})$, and very low birth weight $(<1500 \mathrm{~g})$. Risk ratios with $95 \%$ confidence intervals were estimated with the use of modified Poisson models to adjust for confounding.

RESULTS-There were 4646 birth outcomes. Few infants or fetuses were exposed to TDFFTC-LPV/r (128 [2.8\%]) as the initial ART regimen during gestation, in contrast with TDF-FTCATV/r (539 [11.6\%]) and ZDV-3TC-LPV/r (954 [20.5\%]). As compared with women receiving ZDV-3TC-LPV/r, women receiving TDF-FTC-LPV/r had a similar risk of preterm birth (risk ratio, $0.90 ; 95 \%$ confidence interval [CI], 0.60 to 1.33 ) and low birth weight (risk ratio, $1.13 ; 95 \%$ CI, 0.78 to 1.64). As compared to women receiving TDF-FTC-ATV/r, women receiving TDFFTC-LPV/r had a similar or slightly higher risk of preterm birth (risk ratio, 1.14; 95\% CI, 0.75 to

Address reprint requests to Dr. Patel at the Department of Epidemiology, Harvard T.H. Chan School of Public Health, 677 Huntington Ave., Boston, MA 02115, or to Dr. Rough at ker704@mail.harvard.edu.

Disclosure forms provided by the authors are available with the full text of this article at NEJM.org. 
1.72) and low birth weight (risk ratio, $1.45 ; 95 \% \mathrm{CI}, 0.96$ to 2.17 ). There were no significant differences between regimens in the risk of very preterm birth or very low birth weight.

CONCLUSIONS-The risk of adverse birth outcomes was not higher with TDF-FTC-LPV/r than with ZDV-3TC-LPV/r or TDF-FTC-ATV/r among HIV-infected women and their infants in the United States, although power was limited for some comparisons. (Funded by the National Institutes of Health and others.)

The use of three-drug antiretroviral therapy (ART) during pregnancy has reduced the risk of perinatal transmission of human immunodeficiency virus (HIV) to less than $1 \%, 1,2$ becoming the standard of care in the United States and globally. ${ }^{3,4}$ Although U.S. and World Health Organization (WHO) perinatal guidelines specify which ART regimens are preferred during pregnancy, recommendations are based on a small body of clinical safety data, expert opinion, and programmatic considerations, including regimen harmonization across subpopulations.

Recently, the Promoting Maternal and Infant Survival Everywhere (PROMISE) trial, conducted at multiple sites in sub-Saharan Africa and India, identified potential safety concerns for one ART regimen. ${ }^{2}$ Pregnant women randomly assigned to receive tenofovir disoproxil fumarate, emtricitabine, and ritonavir-boosted lopinavir (TDF- FTC-LPV/r) were more than twice as likely to have infants born very prematurely $(<34$ completed weeks of gestation) or at very low birth weight $(<1500 \mathrm{~g})$ as those assigned to receive zidovudine, lamivudine, and ritonavir-boosted lopinavir (ZDV-3TC-LPV/r). ${ }^{2}$ Infants with in utero exposure to TDF-FTC-LPV/r also had a substantially higher risk of death within 14 days after delivery.

The results of the PROMISE trial were unexpected, given that numerous observational studies had shown the use of TDF-FTC-based regimens during pregnancy to be safe with respect to most infant outcomes. ${ }^{5-18}$ Understanding the safety of in utero exposure to TDFFTC-based regimens is critical, because the WHO recommends a once-daily TDF-FTCbased regimen as first-line therapy for all HIV-infected adults, including pregnant women. ${ }^{4}$ It is unclear whether the risks observed in the PROMISE trial are shared by all TDF-FTCbased regimens or how the findings will translate to other settings. Of particular interest is the safety of TDF-FTC with ritonavir-boosted atazanavir (ATV/r), because it is one of the most commonly used regimens among HIV-infected pregnant women in the United States.

Using data from two large, U.S.-based, perinatal cohort studies, we made three pairwise comparisons examining the risk of adverse birth outcomes among infants with in utero exposure to one of two PROMISE regimens, ZDV-3TC-LPV/r or TDF-FTC-LPV/r, or to a third regimen not studied in the PROMISE trial, TDF- FTC-ATV/r.

\section{METHODS}

\section{STUDY PARTICIPANTS AND DESIGN}

This study used data from two U.S.-based multi-site cohorts of pregnant women with HIV infection and their infants: the Surveillance Monitoring for ART Toxicities (SMARTT) study of the Pediatric HIV/AIDS Cohort Study (PHACS) and the P1025 study of the International 
Maternal Pediatric Adolescent AIDS Clinical Trials (IMPAACT) Network. We included mother-infant pairs enrolled from April 1, 2007, through March 1, 2016, in the Dynamic cohort of the SMARTT study, which enrolled women with HIV infection and their infants at 23 or more weeks of gestation through 1 week after delivery. The P1025 study was active from 2002 through 2013 and enrolled pregnant women from either 8 weeks of gestation (2007-2013) or 14 weeks of gestation (2002-2006) through 14 days after delivery. Detailed descriptions of each study have been published previously. ${ }^{19,20}$

This analysis included all the infants with an observed birth outcome in the SMARTT or P1025 study, when the first ART regimen that their mothers used during pregnancy was one of the three being investigated: TDF-FTC-LPV/r, TDF- FTC-ATV/r, or ZDV-3TC-LPV/r. Because mother- infant pairs could be enrolled in both the SMARTT and P1025 studies, duplicate observations were removed during data pooling. The SMARTT and P1025 protocols were approved by institutional review boards at each participating site. Written informed consent was obtained from all the participating mothers.

\section{ANTIRETROVIRAL EXPOSURE AND CONFOUNDER CLASSIFICATION}

Information on maternal antiretroviral exposure, including regimen start and stop dates, was abstracted from medical records in the SMARTT and P1025 studies. For this study, a participant's antiretroviral exposure was classified as the first regimen taken during pregnancy, mimicking the intention-to-treat principle. We further classified antiretroviral exposure according to the timing of therapy initiation: before pregnancy, during the first trimester, or during the second or third trimester.

Maternal demographic and behavioral characteristics, including age at delivery, education, race or ethnic group, and substance use during pregnancy, were based on participant report. CD4 cell count, HIV viral load, and information on diagnoses (pregestational diabetes, hepatitis B or C virus infection, and sexually transmitted infections) were abstracted from medical charts and laboratory results. Information on parity, previous preterm delivery, and hypertension was not reliably collected in one or both of the included studies.

For participants coenrolled in both the SMARTT and P1025 studies, data from the P1025 study were generally more complete and were prioritized, with several exceptions. Information on substance use was used preferentially from the SMARTT study (in which it was collected more thoroughly), ${ }^{21}$ and if covariate or outcome values were missing in the P1025 study, data from the SMARTT study were used when available.

\section{OUTCOME CLASSIFICATION}

In both the SMARTT and P1025 studies, gestational age was assessed with the use of obstetric estimates based on ultrasonography, physical examination, or the date of the last menstrual period. ${ }^{22,23}$ In accordance with definitions used in the PROMISE trial, deliveries occurring before 37 completed weeks of gestation were considered to be preterm and those occurring before 34 completed weeks to be very preterm. We classified birth weights of less than $2500 \mathrm{~g}$ as low birth weight and those of less than $1500 \mathrm{~g}$ as very low birth weight. We analyzed a composite adverse outcome (preterm birth, low birth weight, fetal loss, or 
neonatal death [ $<14$ days after delivery]) and severe adverse outcome (very preterm birth, very low birth weight, fetal loss, or neonatal death).

\section{STATISTICAL ANALYSIS}

Maternal characteristics were summarized, with stratification according to initial ART regimen during pregnancy. The unadjusted risk of each outcome according to regimen was calculated with the corresponding 95\% Wald confidence intervals.

In primary analyses, we made three separate pairwise comparisons of ART regimens: TDFFTC-LPV/r versus ZDV-3TC-LPV/r (the comparison made in the PROMISE trial), TDFFTC-ATV/r versus ZDV-3TC-LPV/r (a comparison between two common regimens used during pregnancy in the United States), and TDF-FTC-LPV/r versus TDF-FTC-ATV/r (a comparison between two different protease inhibitors used with TDF-FTC). For analyses of gestational age and birth weight, comparisons were limited to live-born infants. Modified Poisson generalized-estimating-equation models were used to estimate risk ratios and corresponding $95 \%$ confidence intervals, with robust variances to account for correlations between multiple-gestation pregnancies and multiple pregnancies. No corrections were made for multiple testing. In adjusted analyses, four risk factors showing consistently strong associations with the outcomes across multiple studies were included in the models (race or ethnic group, smoking status [yes vs. no vs. missing data], diabetes [yes vs. no], and sexually transmitted infection [yes vs. no vs. missing data]). ${ }^{24}$ We also examined the potential confounders listed in Table S1 in the Supplementary Appendix (available with the full text of this article at NEJM.org) and included additional variables associated with both the exposures and outcomes of interest. Owing to the low number of events of very preterm birth, very low birth weight, and severe adverse outcomes, multivariable adjustment was not feasible for these outcomes.

For all outcomes and exposure comparisons, we conducted four subgroup analyses to ensure that findings were robust and to identify potential effect modification: women who initiated ART during pregnancy, women who continued an ART regimen initiated before conception, women with a first singleton pregnancy observed in either of the cohorts, and women who did not switch their regimen during pregnancy. Owing to sample-size limitations, subgroup analyses were not adjusted for potential confounders. The results of all 72 subgroup analyses performed are reported without correction for type I error; 3.6 false positives would be expected by chance.

In secondary analyses, we compared the use of TDF-FTC plus any protease inhibitor with ZDV-3TC plus any protease inhibitor using the same methods described above. We also summarized the risks of our outcomes according to the timing of regimen initiation.

Unless otherwise noted, the unit of analysis for results is the mother-infant pair. All analyses were conducted with the use of SAS software, version 9.4 (SAS Institute). All the authors vouch for the completeness and accuracy of the data and analyses presented. 


\section{RESULTS \\ SAMPLE DESCRIPTION}

Among the 2389 mother-infant pairs enrolled in the Dynamic SMARTT cohort and 3146 enrolled in the P1025 cohort, there were 4646 birth outcomes (live births as well as stillbirths and other recorded fetal deaths) to 3847 unique women (Fig. S1 in the Supplementary Appendix). There were 4480 singleton, 80 twin, and 2 triplet pregnancies. A total of 128 infants or fetuses (2.8\%) were exposed to TDF-FTC-LPV/r as the initial ART regimen during gestation, 539 (11.6\%) were exposed to TDF-FTC-ATV/r, and 954 (20.5\%) were exposed to ZDV-3TC-LPV/r. The percentage of women who switched regimens during pregnancy varied; $47.7 \%$ whose initial regimen was TDF-FTC-LPV/r changed regimens before delivery, as compared with $31.7 \%$ in the TDF- FTC-ATV/r group and $35.6 \%$ in the ZDV-3TC- LPV/r group.

The distribution of most maternal characteristics was similar across the regimens (Table 1, and Table S1 in the Supplementary Appendix). TDF-FTC-ATV/r was used more frequently in later calendar years than the other two regimens, and women who received TDF-FTC$\mathrm{ATV} / \mathrm{r}$ as their initial regimen during pregnancy tended to be older. The timing of regimen initiation also differed among the regimens; $76.3 \%$ of the women whose first regimen was ZDV-3TC-LPV/r initiated therapy in the second or third trimester (as compared with 40.6\% for TDF-FTC-LPV/r and 35.6\% for TDF-FTC-ATV/r), whereas TDF- FTC-based regimens were more likely to be started before conception (45.3\% for TDF-FTC- LPV/r and $49.2 \%$ for TDF-FTC-ATV/r, as compared with $11.6 \%$ for ZDV-3TC-LPV/r). Correspondingly, women who received ZDV-3TC-LPV/r were more likely to receive a diagnosis of HIV infection during pregnancy, and women who received the TDF-FTCbased regimens were more likely to have a first viral-load measurement of less than 400 copies per milliliter.

\section{PRIMARY ANALYSIS}

There were 10 recorded fetal losses: 2 (1.6\%) among women who received TDF-FTC$\mathrm{LPV} / \mathrm{r}$ as their first regimen during pregnancy, $2(0.4 \%)$ among those receiving TDF-FTCATV/r, and $6(0.6 \%)$ among those receiving ZDV-3TC-LPV/r. One infant each in the TDFFTC-ATV/r and ZDV-3TC-LPV/r groups died within 14 days after delivery $(0.2 \%$ and $0.1 \%$, respectively); in both cases, the cause of death was extreme prematurity. Across the regimens, the risk of preterm birth ranged from 16.1 to $21.4 \%$, the risk of low birth weight ranged from 16.2 to $23.8 \%$, and the risk of any adverse outcome ranged from 23.7 to $28.1 \%$ (Table 2). The risks of these outcomes were lowest in the TDF-FTC-ATV/r group, though there were only slight differences between regimens for severe adverse outcomes. In unadjusted analyses, the 95\% confidence intervals for all comparisons of TDF-FTC-LPV/r with ZDV-3TC- LPV/r included the null value of 1 (Table 3). TDF-FTC-ATV/r was associated with a slightly lower risk of preterm birth, low birth weight, and any adverse outcome than TDF-FTC-LPV/r or ZDV-3TC-LPV/r.

After adjustment, the comparison of TDF- FTC-LPV/r with ZDV-3TC-LPV/r yielded estimates close to the null value for preterm birth (risk ratio, $0.90 ; 95 \%$ confidence interval 
[CI], 0.60 to 1.33 ), low birth weight (risk ratio, 1.13 ; $95 \% \mathrm{CI}, 0.78$ to 1.64 ), and any adverse outcome (risk ratio, $0.92 ; 95 \%$ CI, 0.67 to 1.28 ) (Table 3). For the outcomes of preterm birth, low birth weight, and any adverse outcome, TDF-FTC-ATV/r appeared to have lower risks than the LPV/r-based regimens; however, many of these associations were not significant. Secondary analyses comparing TDF-FTC-any protease inhibitor (960 women) with ZDV-3TC-any protease inhibitor (1593 women) showed that TDF-FTC-based regimens were associated with lower risks of preterm birth (risk ratio, $0.77 ; 95 \% \mathrm{CI}, 0.62$ to 0.96) (Tables S2 through S4 in the Supplementary Appendix).

\section{SECONDARY AND SUBGROUP ANALYSES}

Results of subgroup analyses for the outcomes of preterm birth and low birth weight are presented in Figure 1. (For other outcomes, see Figs. S2 through S5 in the Supplementary Appendix.) For all regimen comparisons, subgroup restriction to women with first singleton pregnancies and to women who did not switch regimens during pregnancy did not substantially shift crude or adjusted risk estimates in the overall population. However, among those who initiated therapy before conception, unadjusted risks of preterm birth and low birth weight were higher in the TDF-FTC-LPV/r group than in the TDF- FTC-ATV/r group (risk ratio for preterm birth, 1.51 [95\% CI, 0.89 to 2.55]; risk ratio for low birth weight, 1.97 [95\% CI, 1.15 to 3.38]).

We observed a higher risk of preterm and very preterm birth among women who initiated any of the regimens before pregnancy than among those who initiated regimens in the second or third trimester (Fig. 2). Risks of very low birth weight and severe adverse outcomes were slight ly higher among women initiating ART before pregnancy or in the first trimester than among those initiating ART in the second or third trimester.

\section{DISCUSSION}

In two large, U.S.-based, multisite cohorts of pregnant women with HIV infection and their infants, the use of TDF-FTC-LPV/r during pregnancy was not associated with a significantly higher risk of adverse infant birth outcomes than the use of ZDV-3TC-LPV/r or TDF-FTC-ATV/r, with one exception. In crude subgroup analyses with no adjustment for multiple comparisons, we observed higher risks of preterm birth, low birth weight, and a composite adverse outcome among women initiating TDF-FTC- LPV/r before conception than among those initiating ZDV-3TC-LPV/r or TDF-FTC-ATV/r before conception. Additional analyses comparing TDF- FTC-any protease inhibitor with ZDV-3TC-any protease inhibitor indicated slightly lower risks of preterm birth and any adverse outcome with the TDF-FTC-containing regimens.

We did not observe substantial differences in risks of preterm birth and low birth weight between TDF-FTC-LPV/r and ZDV-3TC-LPV/r, findings consistent with the results of the PROMISE trial. We did not find a higher risk of very preterm birth and very low birth weight with TDF- FTC-LPV/r than with ZDV-3TC-LPV/r, findings contrary to the results of the PROMISE trial. Given the small number of women who received TDF-FTC-LPV/r and the rarity of these outcomes in the sample, some comparisons involving this group had low power to detect a statistically or clinically significant difference. The resulting wide 
95\% confidence intervals around the estimated risk ratios of very preterm birth, very low birth weight, and severe adverse outcomes included the values observed in the PROMISE trial.

Dosing of TDF-FTC-LPV/r may also have differed between the PROMISE trial and the SMARTT and P1025 studies; to compensate for reduced plasma levels observed in pharmacokinetic studies, ${ }^{25-27}$ the PROMISE protocol specified 1.5 times the normal dosing of LPV/r during the third trimester. Because dosing information was not collected in the SMARTT and P1025 studies, it is unclear whether women received similar doses in this study. Another potential explanation for the lack of an association could be the relatively high rate of switching from TDF-FTC- LPV/r before delivery. As in any observational study, residual confounding could explain discrepant findings; yet, for most measured characteristics, there were limited differences among women taking different regimens. Owing to limited sample sizes, we were unable to emulate the PROMISE eligibility criterion of including only women with CD4 counts of more than 350 cells per cubic millimeter before regimen initiation. Finally, there are differences in the characteristics of the participants and the care provided to pregnant women and their infants in the United States as compared with the low-resource settings in which the PROMISE trial was conducted that may have led to differences in our results.

TDF-FTC-LPV/r was rarely used by pregnant women with HIV infection in either U.S.based cohort, and its use in other settings is also limited, because it is not among the firstline regimens recommended by the WHO. ${ }^{4}$ Concerns regarding the use of TDF-FTC-LPV/r during pregnancy remain; further investigation is warranted to understand why women who initiated TDF-FTC- LPV/r before conception had higher risks of preterm birth, low birth weight, and any adverse outcome than women who initiated ZDV-3TC- LPV/r or TDFFTC-ATV/r before conception in subgroup analyses.

The use of ATV/r with TDF-FTC appeared to be associated with a lower risk of adverse infant birth outcomes than the use of the other regimens studied. However, our results must be interpreted alongside other safety findings related to TDF, FTC, and ATV/r use in pregnancy. Several studies have shown relationships between ATV and delayed language development ${ }^{28-30}$ and social- emotional development. ${ }^{30}$ First-trimester ATV use has also been linked to an increased risk of skin and musculoskeletal malformations, ${ }^{16}$ and in utero TDF exposure may be linked to reduced bone mineral content in infants. ${ }^{14}$

Several studies have evaluated the association between the timing of ART initiation and infant birth outcomes; a recent meta-analysis showed increased risks associated with preconception ART for preterm birth, very preterm birth, and low birth weight. ${ }^{31}$ Our study adds to the limited number of studies examining the risks of severe adverse infant birth outcomes according to the timing of regimen initiation. In secondary analyses, we observed higher risks of these outcomes among women who initiated ART before conception or in the first trimester than among those women who initiated regimens in the second or third trimester, findings that suggest that women who initiate ART before the second trimester may require more careful monitoring. 
This study has several important limitations. First, because enrollment late in pregnancy and shortly after delivery was allowed in both the P1025 and SMARTT studies, stillbirths and very preterm births may not be well captured. Al though we do not expect underascertainment to be differential between regimens, the presence of differential measurement could cause selection bias. Second, there was limited information on some important predictors of preterm birth and low birth weight, including parity, previous preterm delivery, and hypertension. Because these variables could not be controlled for in analyses, estimates could be confounded if the distribution of these predictors varied according to ART regimen. Third, because of the rare occurrence of severe adverse outcomes in the sample, some comparisons, especially those involving TDF- FTC-LPV/r, had limited power to detect safety signals. Finally, it is unclear how generalizable our findings are outside the United States; interactions among host genetics, the pharmacokinetics of antiretroviral drugs, and the risks of specific outcomes have been reported, which may modify associations observed in different contexts..$^{32-34}$

In conclusion, we did not observe a higher risk of adverse or severe adverse birth outcomes among infants with in utero exposure to TDF- FTC-LPV/r than among those exposed to ZDV-3TC-LPV/r or TDF-FTC-ATV/r. This regimen was also infrequently used in our cohort of U.S. women with HIV infection relative to the other regimens studied. Our findings also suggest that the use of TDF-FTC-ATV/r during pregnancy appears to carry similar or lower risks of preterm birth and low birth weight than the use of ZDV-3TC$\mathrm{LPV} / \mathrm{r}$.

\section{Supplementary Material}

Refer to Web version on PubMed Central for supplementary material.

\section{Acknowledgments}

The conclusions and opinions expressed in this article are those of the authors and do not necessarily reflect those of the National Institutes of Health or U.S. Department of Health and Human Services. The funders had no role in the design and conduct of the study; collection, management, analysis, and interpretation of the data; and preparation, review, or approval of the manuscript.

The Pediatric HIV/AIDS Cohort Study (PHACS) was supported by the Eunice Kennedy Shriver National Institute of Child Health and Human Development (NICHD) with cofunding from the National Institute on Drug Abuse, the National Institute of Allergy and Infectious Diseases (NIAID), the Office of AIDS Research, the National Institute of Mental Health (NIMH), the National Institute of Neurological Disorders and Stroke, the National Institute on Deafness and Other Communication Disorders, the National Heart, Lung, and Blood Institute, the National Institute of Dental and Craniofacial Research, and the National Institute on Alcohol Abuse and Alcoholism, through cooperative agreements with the Harvard T.H. Chan School of Public Health (HD052102) (principal investigator, George Seage; project director, Julie Alperen) and the Tulane University School of Medicine (HD052104) (principal investigator, Russell Van Dyke; coprincipal investigator, Ellen Chadwick; project director, Patrick Davis). Data-management services were provided by the Frontier Science and Technology Research Foundation (principal investigator, Suzanne Siminski), and regulatory services and logistical support were provided by Westat (principal investigator, Julie Davidson). Overall support for the International Maternal Pediatric Adolescent AIDS Clinical Trials (IMPAACT) Network was provided by the NIAID of the National Institutes of Health (NIH) under award numbers UM1AI068632 (IMPAACT LOC), UM1AI068616 (IMPAACT SDMC), and UM1AI106716 (IMPAACT LC), with co-funding from the NICHD and the NIMH. Dr. Rough was additionally supported by grant number T32 AI007433 from the NIAID and a training grant from the Pharmacoepidemiology Concentration at the Harvard T.H. Chan School of Public Health. 
We thank the children and families for their participation in the PHACS Surveillance Monitoring for ART Toxicities (SMARTT) study and the IMPAACT P1025 study, and the persons and institutions involved in the conduct of the PHACS SMARTT study and the IMPAACT P1025 study.

\section{References}

1. Cooper ER, Charurat M, Mofenson L, et al. Combination antiretroviral strategies for the treatment of pregnant HIV-1-infected women and prevention of perinatal HIV-1 transmission. J Acquir Immune Defic Syndr. 2002; 29:484-94. [PubMed: 11981365]

2. Fowler MG, Qin M, Fiscus SA, et al. Benefits and risks of antiretroviral therapy for perinatal HIV prevention. N Engl J Med. 2016; 375:1726-37. [PubMed: 27806243]

3. AIDSinfo. Recommendations for the use of antiretroviral drugs in pregnant women with HIV infection and interventions to reduce perinatal HIV transmission in the United States. Washington, DC: Department of Health and Human Services; 2017. https://aidsinfo.nih.gov/guidelines/html/3/ perinatal-guidelines/0

4. Consolidated guidelines on the use of antiretroviral drugs for treating and preventing HIV infection: recommendations for a public health approach. second. Geneva: World Health Organization; 2016. http://www.who.int/hiv/pub/arv/arv-2016/en/

5. Nurutdinova D, Onen NF, Hayes E, Mondy K, Overton ET. Adverse effects of tenofovir use in HIVinfected pregnant women and their infants. Ann Pharmacother. 2008; 42:1581-5. [PubMed: 18957630]

6. Kinai E, Hosokawa S, Gomibuchi H, Gatanaga H, Kikuchi Y, Oka S. Blunted fetal growth by tenofovir in late pregnancy. AIDS. 2012; 26:2119-20. [PubMed: 23052356]

7. Viganò A, Mora S, Giacomet V, et al. In utero exposure to tenofovir disoproxil fumarate does not impair growth and bone health in HIV-uninfected children born to HIV-infected mothers. Antivir Ther. 2011; 16:1259-66. [PubMed: 22155907]

8. Siberry GK, Williams PL, Mendez H, et al. Safety of tenofovir use during pregnancy: early growth outcomes in HIV-exposed uninfected infants. AIDS. 2012; 26:1151-9. [PubMed: 22382151]

9. Gibb DM, Kizito H, Russell EC, et al. Pregnancy and infant outcomes among HIV-infected women taking long-term ART with and without tenofovir in the DART trial. PLoS Med. 2012; 9(5):e1001217. [PubMed: 22615543]

10. Ransom CE, Huo Y, Patel K, et al. Infant growth outcomes after maternal tenofovir disoproxil fumarate use during pregnancy. J Acquir Immune Defic Syndr. 2013; 64:374-81. [PubMed: 24169122]

11. Celen MK, Mert D, Ay M, et al. Efficacy and safety of tenofovir disoproxil fumarate in pregnancy for the prevention of vertical transmission of HBV infection. World J Gastroenterol. 2013; 19:9377-82. [PubMed: 24409065]

12. Greenup AJ, Tan PK, Nguyen V, et al. Efficacy and safety of tenofovir disoproxil fumarate in pregnancy to prevent perinatal transmission of hepatitis B virus. J Hepatol. 2014; 61:502-7. [PubMed: 24801414]

13. Vivanti A, Soheili TS, Cuccuini W, et al. Comparing genotoxic signatures in cord blood cells from neonates exposed in utero to zidovudine or tenofovir. AIDS. 2015; 29:1319-24. [PubMed: 25513819]

14. Siberry GK, Jacobson DL, Kalkwarf HJ, et al. Lower newborn bone mineral content associated with maternal use of tenofovir disoproxil fumarate during pregnancy. Clin Infect Dis. 2015; 61:996-1003. [PubMed: 26060285]

15. Knapp KM, Brogly SB, Muenz DG, et al. Prevalence of congenital anomalies in infants with in utero exposure to anti-retrovirals. Pediatr Infect Dis J. 2012; 31:164-70. [PubMed: 21983213]

16. Williams PL, Crain MJ, Yildirim C, et al. Congenital anomalies and in utero antiretroviral exposure in human immunodeficiency virus-exposed uninfected infants. JAMA Pediatr. 2015; 169:48-55. [PubMed: 25383770]

17. Brogly SB, Abzug MJ, Watts DH, et al. Birth defects among children born to human immunodeficiency virus-infected women: pediatric AIDS clinical trials protocols 219 and 219C. Pediatr Infect Dis J. 2010; 29:721-7. [PubMed: 20539252] 
18. Sibiude J, Mandelbrot L, Blanche S, et al. Association between prenatal exposure to antiretroviral therapy and birth defects: an analysis of the French perinatal cohort study (ANRS CO1/CO11). PLoS Med. 2014; 11(4):e1001635. [PubMed: 24781315]

19. Williams PL, Seage GR III, Van Dyke RB, et al. A trigger-based design for evaluating the safety of in utero antiretroviral exposure in uninfected children of human immunodeficiency virus-infected mothers. Am J Epidemiol. 2012; 175:950-61. [PubMed: 22491086]

20. Livingston EG, Huo Y, Patel K, Tuomala RE, Scott GB, Stek A. Complications and route of delivery in a large cohort study of HIV-1-infected women - IMPAACT P1025. J Acquir Immune Defic Syndr. 2016; 73:74-82. [PubMed: 27082506]

21. Tassiopoulos K, Read JS, Brogly S, et al. Substance use in HIV-infected women during pregnancy: self-report versus meconium analysis. AIDS Behav. 2010; 14:1269-78. [PubMed: 20532607]

22. Patel K, Shapiro DE, Brogly SB, et al. Prenatal protease inhibitor use and risk of preterm birth among HIV-infected women initiating antiretroviral drugs during pregnancy. J Infect Dis. 2010; 201:1035-44. [PubMed: 20196654]

23. Watts DH, Williams PL, Kacanek D, et al. Combination antiretroviral use and preterm birth. J Infect Dis. 2013; 207:612-21. [PubMed: 23204173]

24. Goldenberg RL, Culhane JF, Iams JD, Romero R. Epidemiology and causes of preterm birth. Lancet. 2008; 371:75-84. [PubMed: 18177778]

25. Stek AM, Mirochnick M, Capparelli E, et al. Reduced lopinavir exposure during pregnancy. AIDS. 2006; 20:1931-9. [PubMed: 16988514]

26. Mirochnick M, Best BM, Stek AM, et al. Lopinavir exposure with an increased dose during pregnancy. J Acquir Immune Defic Syndr. 2008; 49:485-91. [PubMed: 18989231]

27. Best BM, Stek AM, Mirochnick M, et al. Lopinavir tablet pharmacokinetics with an increased dose during pregnancy. J Acquir Immune Defic Syndr. 2010; 54:381-8. [PubMed: 20632458]

28. Sirois PA, Huo Y, Williams PL, et al. Safety of perinatal exposure to antiretroviral medications: developmental outcomes in infants. Pediatr Infect Dis J. 2013; 32:648-55. [PubMed: 23340561]

29. Rice ML, Zeldow B, Siberry GK, et al. Evaluation of risk for late language emergence after in utero antiretroviral drug exposure in HIV-exposed uninfected infants. Pediatr Infect Dis J. 2013; 32(10):e406-e413. [PubMed: 24067563]

30. Caniglia EC, Patel K, Huo Y, et al. Atazanavir exposure in utero and neurodevelopment in infants: a comparative safety study. AIDS. 2016; 30:1267-78. [PubMed: 26867136]

31. Uthman OA, Nachega JB, Anderson J, et al. Timing of initiation of antiretroviral therapy and adverse pregnancy outcomes: a systematic review and meta-analysis. Lancet HIV. 2017; 4(1):e21e30. [PubMed: 27864000]

32. Elens L, Vandercam B, Yombi J-C, Lison D, Wallemacq P, Haufroid V. Influence of host genetic factors on efavirenz plasma and intracellular pharmacokinetics in HIV-1-infected patients. Pharmacogenomics. 2010; 11:1223-34. [PubMed: 20860463]

33. López Aspiroz E, Cabrera Figueroa SE, Porras Hurtado GL, Cruz Guerrero R, Domínguez-Gil Hurlé A, Carracedo A. Pharmacogenetic analysis of SNPs in genes involved in the pharmacokinetics and response to lopinavir/ritonavir therapy. Curr Drug Metab. 2013; 14:729-37. [PubMed: 24001122]

34. Purswani MU, Patel K, Winkler CA, et al. Brief report: APOL1 renal risk variants are associated with chronic kidney disease in children and youth with perinatal HIV infection. J Acquir Immune Defic Syndr. 2016; 73:63-8. [PubMed: 27035887] 


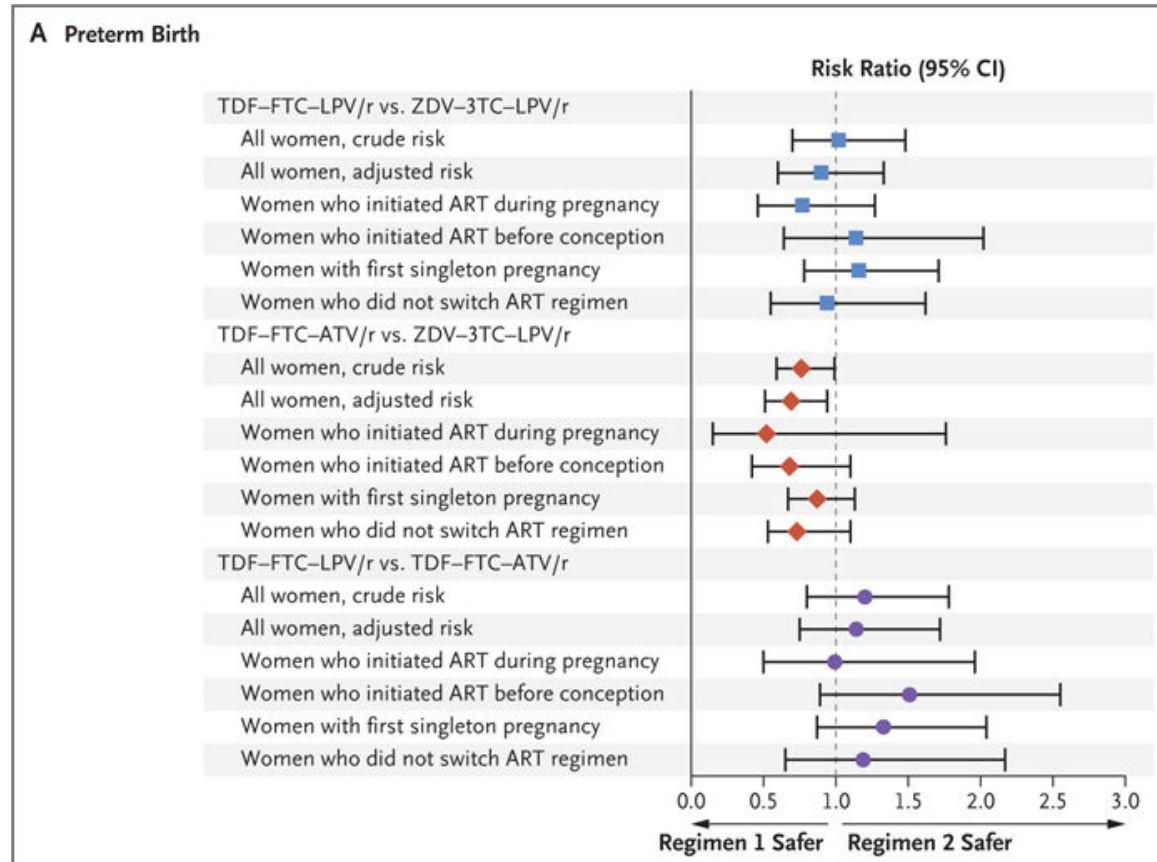

B Low Birth Weight

TDF-FTC-LPV/rvs. ZDV-3TC-LPV/r

All women, crude risk

All women, adjusted risk

Women who initiated ART during pregnancy

Women who initiated ART before conception

Women with first singleton pregnancy

Women who did not switch ART regimen TDF-FTC-ATV/rvs. ZDV-3TC-LPV/r

All women, crude risk

All women, adjusted risk

Women who initiated ART during pregnancy

Women who initiated ART before conception

Women with first singleton pregnancy

Women who did not switch ART regimen

TDF-FTC-LPV/r vs. TDF-FTC-ATV/r

All women, crude risk

All women, adjusted risk

Women who initiated ART during pregnancy

Women who initiated ART before conception

Women with first singleton pregnancy

Women who did not switch ART regimen

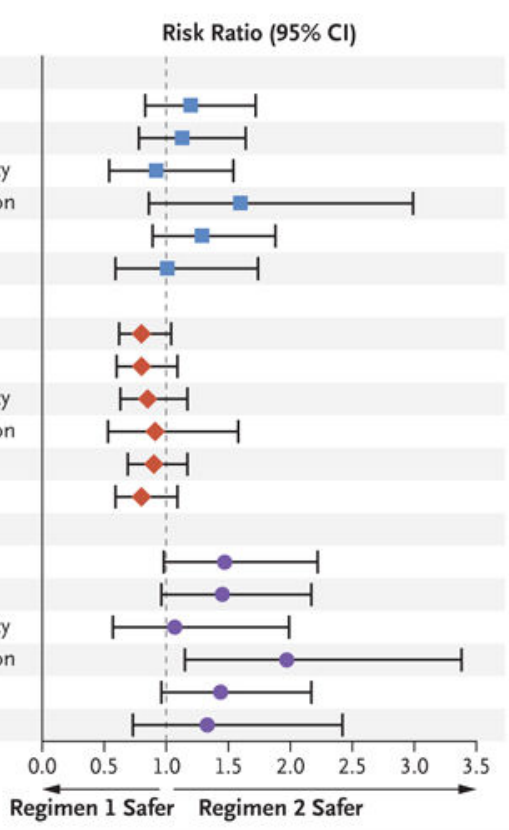

Figure 1. Subgroup Analyses for Comparison of Initial Antiretroviral Regimen during Pregnancy and Risk of Preterm Birth and Low Birth Weight

Preterm birth was defined as delivery before 37 completed weeks of gestation. Low birth weight was defined as a weight of less than $2500 \mathrm{~g}$ at birth. Adjusted risk ratios were obtained from modified Poisson models that were adjusted for race or ethnic group, smoking status (yes vs. no vs. missing data), diabetes (yes vs. no), sexually transmitted infection (yes vs. no vs. missing data), and timing of antiretroviral therapy (ART) initiation (before conception vs. first trimester vs. second or third trimester). 3TC denotes lamivudine, ATV/r 
ritonavir-boosted atazanavir, FTC emtricitabine, LPV/r ritonavir-boosted lopinavir, TDF tenofovir disoproxil fumarate, and ZDV zidovudine. 


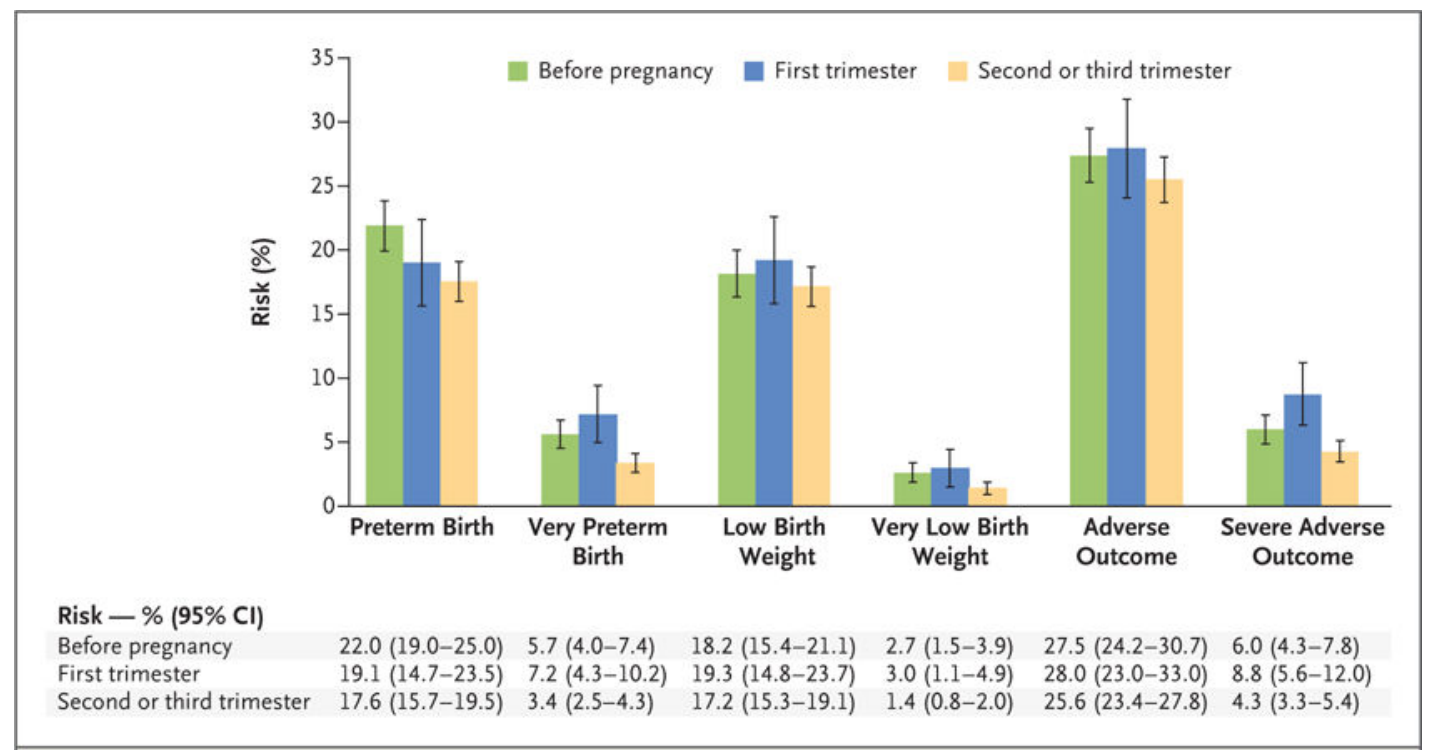

Figure 2. Risk of Birth Outcomes According to Timing of ART initiation for Any of the Three Regimens

Preterm birth was defined as delivery before 37 completed weeks of gestation. Very preterm birth was defined as delivery before 34 completed weeks of gestation. Low birth weight was defined as a weight of less than $2500 \mathrm{~g}$ at birth. Very low birth weight was defined as a weight of less than $1500 \mathrm{~g}$ at birth. An adverse outcome was defined as preterm birth, low birth weight, fetal loss, or neonatal death $(<14$ days after delivery). A severe adverse outcome was defined as very preterm birth, very low birth weight, fetal loss, or neonatal death ( $<14$ days after delivery). I bars indicate $95 \%$ confidence intervals. 
Table 1

Select Maternal Characteristics According to Initial ART Regimen during Pregnancy. ${ }^{*}$

\begin{tabular}{|c|c|c|c|}
\hline Characteristic & $\begin{array}{l}\text { TDF-FTC-LPV/r } \\
(\mathbf{N}=128)\end{array}$ & $\begin{array}{l}\text { TDF-FTC-ATV/r } \\
(\mathbf{N}=539) \\
\text { number (percent) }\end{array}$ & $\begin{array}{l}\text { ZDV-3TC-LPV/r } \\
(\mathbf{N}=954)\end{array}$ \\
\hline \multicolumn{4}{|l|}{ Year of delivery } \\
\hline $2002-2004$ & 0 & 0 & $29(3.0)$ \\
\hline $2005-2008$ & $38(29.7)$ & $92(17.1)$ & $260(27.3)$ \\
\hline 2009-2012 & $76(59.4)$ & $290(53.8)$ & $554(58.1)$ \\
\hline 2013-2016 & $14(10.9)$ & $157(29.1)$ & $111(11.6)$ \\
\hline \multicolumn{4}{|l|}{ Age at delivery } \\
\hline$<24 \mathrm{yr}$ & $50(39.1)$ & $136(25.2)$ & $355(37.2)$ \\
\hline $25-34 \mathrm{yr}$ & $67(52.3)$ & $293(54.4)$ & $473(49.6)$ \\
\hline$>35 \mathrm{yr}$ & $11(8.6)$ & $109(20.2)$ & $125(13.1)$ \\
\hline Missing data & 0 & $1(02)$ & $1(01)$ \\
\hline \multicolumn{4}{|l|}{ Education } \\
\hline Not a high school graduate & $34(26.6)$ & $188(34.9)$ & $331(34.7)$ \\
\hline High school graduate & $61(47.7)$ & $240(44.5)$ & $427(44.8)$ \\
\hline College or more & $33(25.8)$ & $109(20.2)$ & $194(20.3)$ \\
\hline Missing data & 0 & $2(0.4)$ & $2(0.2)$ \\
\hline \multicolumn{4}{|l|}{ Race or ethnic group ${ }^{\dagger}$} \\
\hline Non-Hispanic white & $15(11.7)$ & $44(8.2)$ & $68(7.1)$ \\
\hline Non-Hispanic black & $81(63.3)$ & $365(67.7)$ & $611(64.0)$ \\
\hline Hispanic & $30(23.4)$ & $120(22.3)$ & $258(27.0)$ \\
\hline Other & $1(0.8)$ & $9(17)$ & $11(12)$ \\
\hline Missing data & $1(0.8)$ & $1(02)$ & $6(0.6)$ \\
\hline \multicolumn{4}{|l|}{ First CD4 count in pregnancy } \\
\hline$<250$ cells $/ \mathrm{mm}^{3}$ & $30(23.4)$ & $100(18.6)$ & $194(20.3)$ \\
\hline $250-500$ cells $/ \mathrm{mm}^{3}$ & $47(36.7)$ & $205(38.0)$ & $381(39.9)$ \\
\hline$>500$ cells $/ \mathrm{mm}^{3}$ & $47(36.7)$ & $225(41.7)$ & $365(38.3)$ \\
\hline Missing data & $4(3.1)$ & $9(1.7)$ & $14(1.5)$ \\
\hline \multicolumn{4}{|l|}{ First viral RNA level in pregnancy } \\
\hline$<400$ copies $/ \mathrm{ml}$ & $61(47.7)$ & $277(51.4)$ & $281(29.5)$ \\
\hline $400-10,000$ copies $/ \mathrm{ml}$ & $33(25.8)$ & $137(25.4)$ & $361(37.8)$ \\
\hline$>10,000$ copies $/ \mathrm{ml}$ & $33(25.8)$ & $122(22.6)$ & $305(32.0)$ \\
\hline Missing data & $1(0.8)$ & $3(0.6)$ & $7(0.7)$ \\
\hline \multicolumn{4}{|l|}{ Timing of regimen initiation } \\
\hline Before pregnancy & $58(45.3)$ & $265(49.2)$ & $111(11.6)$ \\
\hline During first trimester & $18(14.1)$ & $82(15.2)$ & $115(12.1)$ \\
\hline During second or third trimester & $52(40.6)$ & $192(35.6)$ & $728(76.3)$ \\
\hline
\end{tabular}

NEngl J Med. Author manuscript; available in PMC 2018 October 26. 


\begin{tabular}{|llll|}
\hline Characteristic & $\begin{array}{l}\text { TDF-FTC-LPV/r } \\
(\mathbf{N = 1 2 8})\end{array}$ & $\begin{array}{l}\text { TDF-FTC-ATV/r } \\
(\mathbf{N = 5 3 9 )} \\
\text { number }(\text { percent })\end{array}$ & $\begin{array}{l}\text { ZDV-3TC-LPV/r } \\
(\mathbf{N}=954)\end{array}$ \\
\hline Alcohol use during pregnancy & & & \\
\hline Yes & $25(19.5)$ & $92(17.1)$ & $182(19.1)$ \\
\hline No & $91(71.1)$ & $432(80.1)$ & $705(73.9)$ \\
\hline Missing data & $12(9.4)$ & $15(2.8)$ & $67(7.0)$ \\
\hline Tobacco use during pregnancy & & & $182(19.1)$ \\
\hline Yes & $30(23.4)$ & $105(19.5)$ & $628(65.8)$ \\
\hline No & $77(60.2)$ & $387(71.8)$ & $144(15.1)$ \\
\hline Missing data & $21(16.4)$ & $47(8.7)$ & $115(12.1)$ \\
\hline Illicit-drug use during pregnancy & & & $687(72.0)$ \\
\hline Yes & $21(16.4)$ & $61(11.3)$ & $152(15.9)$ \\
\hline No & $85(66.4)$ & $427(79.2)$ & \\
\hline Missing data & $22(17.2)$ & $51(9.5)$ & \\
\hline
\end{tabular}

* Mothers may not be unique; some women had multiple pregnancies under study observation. The unit of analysis in this table is the mother-infant pair. Women are classified according to the initial antiretroviral therapy (ART) regimen received during pregnancy. Women who switched regimens are included in these totals. Percentages may not total 100 because of rounding. 3TC denotes lamivudine, ATV/r ritonavir-boosted atazanavir, FTC emtricitabine, LPV/r ritonavir-boosted lopinavir, TDF tenofovir disoproxil fumarate, and ZDV zidovudine.

${ }^{\dagger}$ Race and ethnic group were reported by the patient. 


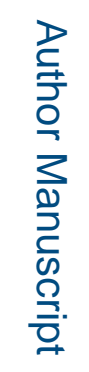



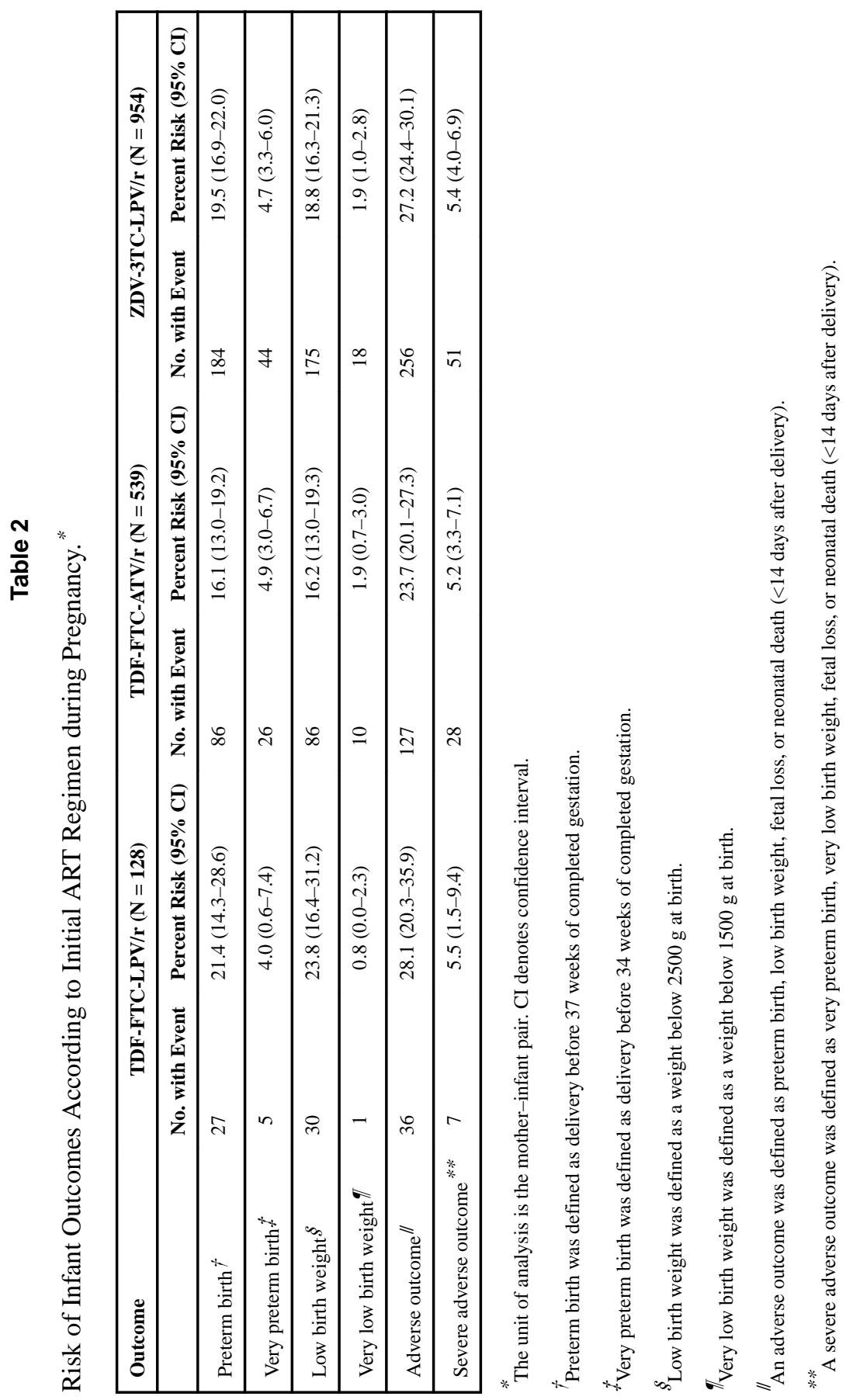

P

?

NEngl J Med. Author manuscript; available in PMC 2018 October 26. 


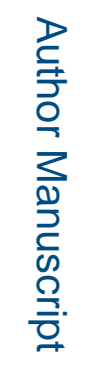

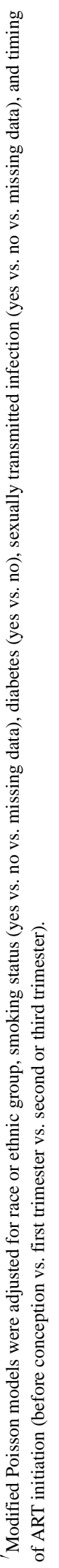

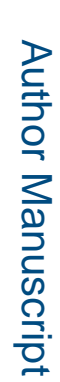

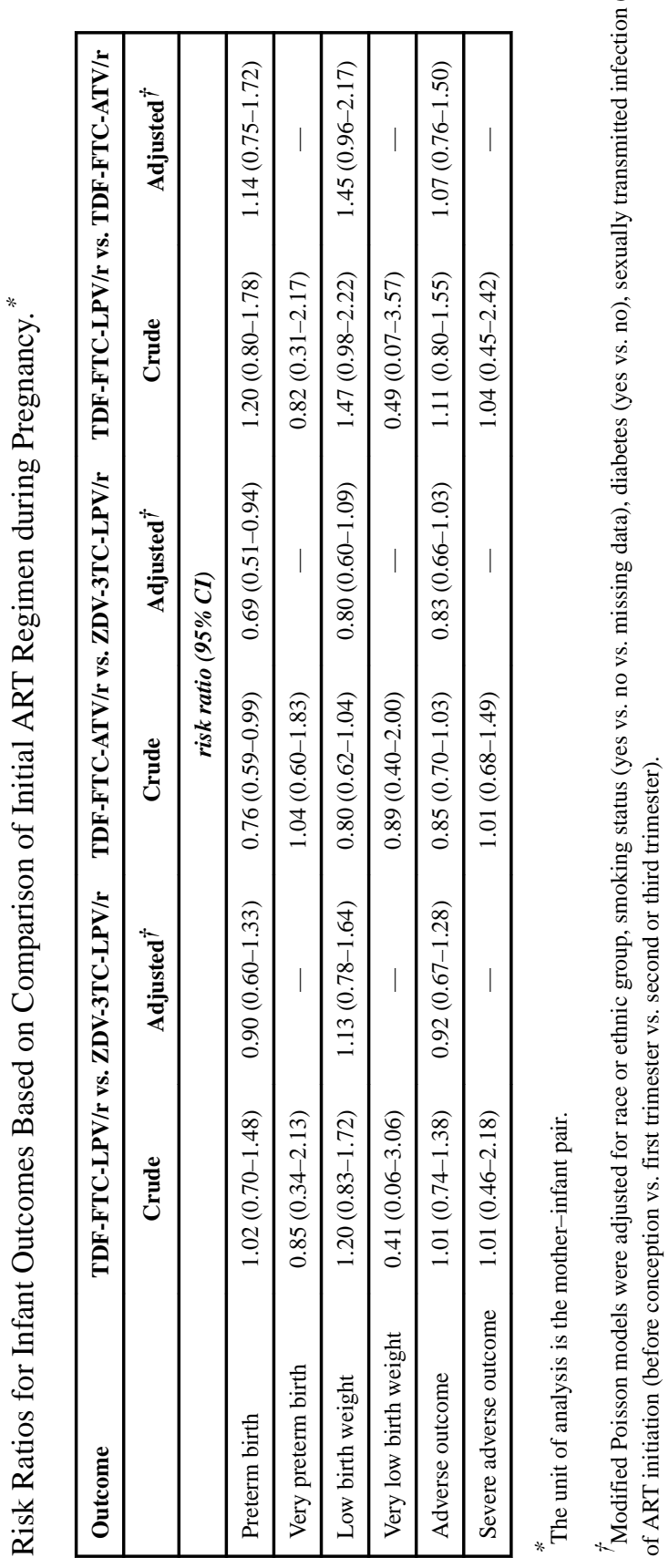

NEngl J Med. Author manuscript; available in PMC 2018 October 26. 